BULLETIN OF THE

AMERICAN MATHEMATICAL SOCIETY

Volume 78, Number 4, July 1972

\title{
AN INVERSE PROBLEM FOR GAUSSIAN PROCESSES1
}

\author{
BY F. ALBERTO GRƯNBAUM
}

Communicated by P. D. Lax, January 11, 1972

Let $X(t)$ be a centered stationary Gaussian process (c.s.G.p.). Its statistics are completely determined by its correlation function

$$
R(s)=E(X(t) X(t+s)) .
$$

This is a positive definite function and we assume it is continuous at the origin.

A problem often considered in the electrical engineering literature is that of determining the statistics of

$$
Y(t)=X^{2}(t) .
$$

The best results, to our knowledge, consist of the computation of some few moments of higher order [1].

Two problems are considered in this note.

1. Does there exist a universal constant $m$ such that the moments of order $\leqq m$ of $Y(t)$ are enough to determine its statistics? (Recall that $m=2$ for a Gaussian process.)

2. How much of the statistics of $X(t)$ can you read off from those of $Y(t)$ ?

The answers to 1 and 2 are embodied in the next two statements.

THEOREM I. Let $m$ be an arbitrary positive integer. There exists a centered stationary Gaussian process $X(t)$ such that the moments of order $\leqq m$ of $Y(t)=X^{2}(t)$ do not suffice to determine $Y$ 's statistics.

THEOREM II. The statistics of $Y(t)=X^{2}(t)$ determine uniquely those of $X(t)$.

The proof of this second result appears in [2]. Stationarity can be disposed of, but the Gaussian character of the process is essential. Finally the real line as a parameter space can be replaced by any arcwise connected space.

ProOf OF Theorem I. A simple computation shows that knowing all the moments of order $\leqq m$ of $Y(t)$ is equivalent to knowing the expressions

$$
\sum_{\pi} R\left(t_{\pi 1}-t_{\pi n}\right) R\left(t_{\pi 2}-t_{\pi 1}\right) \ldots R\left(t_{\pi n}-t_{\pi(n-1)}\right), \quad 2 \leqq n \leqq m .
$$

AMS 1970 subject classifications. Primary 60G15; Secondary 42A88, 43A35, 62M10.

Key words and phrases. Square of a Gaussian process, moments, statistics, uniqueness.

1 The research reported in this paper was supported by the U.S. Atomic Energy Commission, Contract No. AT(30-1)-1480. 
Here $t_{1} \ldots t_{n}$ are arbitrary real numbers, and the sum ranges over the group of all permutations $\pi$ of $n$ elements.

Consider now the function

$$
f(x)=x^{2}(1-\cos x)(1+\varepsilon \cos k x), \quad|\varepsilon|<1, k \geqq 2 .
$$

Its Fourier transform is the positive definite function

$$
\begin{aligned}
R(s) & =\frac{1}{2} \varepsilon(1-|s-k|) & & \text { for }|s-k| \leqq 1, \\
& =1-|s| & & \text { for }|s| \leqq 1, \\
& =\frac{1}{2} \varepsilon(1-|s+k|) & & \text { for }|s+k| \leqq 1, \\
& =0 & & \text { otherwise. }
\end{aligned}
$$

Take $X(t)$ to be a c.s.G.p. with this correlation function. We claim that, by choosing $k$ appropriately, we can conclude that the information contained in (1) is not enough to determine the sign of $\varepsilon$. Indeed, the only arrangements of $t$ 's that give a nonzero contribution to (1) are those for which all the differences $\left|t_{\pi i}-t_{\pi(i-1)}\right|$ are either smaller than 2 or else between $k-1$ and $k+1$. This plus the fact that

$$
\left(t_{\pi 1}-t_{\pi n}\right)+\cdots+\left(t_{\pi n}-t_{\pi(n-1)}\right)=0
$$

implies that for $k>2 m \geqq 2 n$ the number of terms in this sum which are close to $k$ has to match the number of terms which are close to $-k$. But going to the corresponding term in (1) this means that $\varepsilon$ enters with an even power and its sign is lost.

Briefly, for any given $m$ we construct a c.s.G.p. $X(t)$ such that the $m$ order statistics of $Y(t)=X^{2}(t)$ do not determine the correlation of $X(t)$. The proof is now finished if one invokes Theorem II.

It is a pleasure to thank H. P. McKean who suggested a related problem.

\section{REFERENCES}

1. R. V. Deutsch, Nonlinear transformations of random processes, Prentice-Hall Internat. Series in Appl. Math., Prentice-Hall, Englewood Cliffs, N.J., 1962. MR 26 \#6006.

2. F. Alberto Grünbaum, The square of a Gaussian process, Z. Wahrscheinlichkeitstheorie (1972).

Courant Institute of Mathematical Sciences, New York University, New York, NEW YORK 10012 